\title{
Taylor series method in TDOA approach for indoor positioning system
}

\author{
Diniya Jose ${ }^{1}$, Shoney Sebastian ${ }^{2}$ \\ ${ }^{1}$ Department of Computer Science, CHRIST (Deemed to be University), India \\ ${ }^{2}$ Associate Professor, Department of Computer Science, CHRIST (Deemed to be University), India
}

\begin{abstract}
Article Info
Article history:

Received Oct 24, 2018

Revised Apr 15, 2019

Accepted Apr 27, 2019

\section{Keywords:}

Indoor positioning system Taylor series method Time difference of arrival

ABSTRACT

Localisation technologies have always remained in the limelight of positioning-science as researchers have ever shown keen interest to know the exact positions of things. Ultrasonic sensors are mainly used for localisation of mobile robots since they provide high accuracy. This paper presents Taylor Series Method in Time Difference of Arrival approach using ultrasonic sensors.Signals are send from the sensors periodically. The time difference of arrival of signals from the ultrasonic sensors is used by the receiver unit to estimate the location of the mobile unit. The equations formed by using Time Difference of Approach are solved using Taylor Series Method. Taylor Series Method provides a more accurate result since they give less error compared to other methods and they ignore the measurement errors.
\end{abstract} Ultrasonic signals

Copyright () 2019 Institute of Advanced Engineering and Science. All rights reserved.

\section{Corresponding Author:}

Diniya Jose,

Department of Compurt Science,

CHRIST (Deemed to be University),

Hosur Road, Bengaluru-560029, Karnataka, India.

Email: diniya.jose@mca.christuniversity.in

\section{INTRODUCTION}

The process of finding the location of an object or a person is known as positioning [1]. The two types of positioning patterns are indoor positioning and outdoor positioning which depends upon the environment in which it is performed. In outdoor, GPS is one of the most commonly used technologies for positioning. However, GPS does not work inside the building mainly due to three reasons [2]. For GPS to work well, it needs Line Of Sight (LOS) within the GPS satellites and the receivers. The second reason is the building materials. It can pass easily through materials like glass but not through materials like roofs, walls etc. The third reason is that GPS signal comes under category of UHF (Ultra High Frequency) signal band. So there can be several sources of UHF interferences that makes the GPS signals to not work properly.

The technologies used for indoor positioning are RFID [3], Bluetooth [4], Wi-Fi [5], Ultrasound [6], VLC [7] etc. Several characteristics are used for evaluating the performance of an Indoor Positioning System (IPS) [1]. They are accuracy, precision, update interval, coverage, computational cost, offline computing, localisation time and infrastructure.IPS has several applications in our real life [1,8]. They help in the navigation of visually impaired and blind people in public buildings like malls and museums. Navi gation of tourists in places like museums is made easy through IPSs. Another important application of IPS is that they provide medical care in hospitals. They helps in tracking of patients, and even helps to prevent the theft of costly equipment in hospitals. Firefighters and police use IPSs for protection operations. Even the trained police dogs can be tracked which helps in detecting explosives in the buildings. IPSs are used in supermarkets for tracking of customers and commodities. They help in guiding to customers for navigating inside the supermarkets. 
Indoor positioning technologies can be classified in several ways [1]. They can be classified on the basis of need for hardware. That is, based on technologies that contain all the necessary hardware within it and technologies that require additional hardware for positioning inside the building. Classification can be done based on existence of network. That is, the positioning strategies can be classified into network-based technologies and non-network-based technologies. Based on the system architecture the indoor positioning technologies can be classified into three types. They are infrastructure positioning architecture, self-positioning architecture and self-oriented infrastructure-assisted architecture. In infrastructure positioning architecture the location of the object is obtained within the coverage area. Objects calculate the position by themselves in self positioning architecture. However, self-oriented infrastructure-assisted architecture mainly depends on the system that estimates the position. Based on the medium used for positioning classification can be done into ultrasound, magnetic, radio frequency, vision-based and audible sound technologies. Indoor positioning technologies can classified into indoor pedestrian positioning and fixed indoor positioning system. Depending on the available prior knowledge IPS can be classified into parametric and non parametric technologies.Classification is also done based on the type of sensor like camera, RFID, WLAN and Wi-Fi, infrared etc.

In contrast to the above classifications, indoor positioning technologies can be categorized depending on the infrastructure used by the system [1]. They can be classified into building dependent and building independent. Building dependent indoor positioning technologies can be classified based on technologies that use building's infrastructure and technologies that use dedicated infrastructure. Wi-Fi, Bluetooth and Cellular based are the technologies that use building's infrastructure. Dedicated infrastructure is used by technologies like RFID, Zigbee, Ultrasonic and Infrared. Building independent indoor positioning technologies can be classified into Dead Reckoning and Image-based technologies. Dead reckoning locates current position based on the past speed, direction and position. Image-based technologies depend on cameras and other vision-based technologies. Imaged-based technologies can be either building dependent or building independent. Image-based technologies that are building dependent mainly rely on a map of the building or special signs of the buildings. However, image-based technologies that are building independent do not depend on any signs or map of the building.

Mobile Robot Indoor Positioning System has large number of applications in our day to day life like transportation, assistance during the time of surgeries etc. Apart from this, they help in various military activities like scouting and anti-terrorism. However, existing indoor positioning system using mobile robots face the problem of poor positioning accuracy. Therefore inorder to overcome this, Taylor Series Method in Time Difference of Arrival approach using five sensor coordinates is used in this paper which provides a more accurate result.

\section{RELATED WORK}

Different measurement approaches are used in Indoor Positioning System [9]. They are Received Signal Strength Indicator (RSSI), Time of Arrival (TOA), Time Difference of Arrival (TDOA) and Angle of Arrival (AOA). In RSSI, when the signals travel from transmitter to the receiver, they get attenuated. Greater is the chance of attenuation as the distance gets longer. Therefore the distance within the transmitter and the receiver is calculated by using the strength of signal received. Distance can be estimated from information like transmitted signal power, received signal strength, path loss model etc.TOA is the most commonly used technique. In this method the exact time at which the signals are sent from the transmitters and the exact time at which the signals reach the receiver is required. TDOA is the second most commonly used technique. This is the time difference the signal takes to arrive from transmitters to receivers. Here it requires only the time at which the signal arrives the receiver. Compared to TOA the possibility of occurrence of error is less and accuracy of the result obtained is more .AOA is the direction of arrival of signals at the receiver from the transmitters. The direction can be obtained by measuring the angle between the transmitters and receivers. The method is not that widely used since this requires the installation special antennas. Based on the geometry of the transmitter nodes and receiver nodes, the positioning accuracy can be increased [10]. In order to have better accuracy, Position Dilution of Precision (PDOP) value should be less. PDOP is the error in position due to the geometry. It can be written as the square root of HDOP square plus VDOP square. HDOP is the Horizontal Dilution of Precision and VDOP is the Vertical Dilution of Precision. HDOP is the square root LaDOP (Latitude Dilution of Precision) square plus LoDOP (Longitude Dilution of Precision) square.

Ultrasonic sensors are widely used for positioning because of its low cost and better accuracy it provides. Ultrasonic Indoor Positioning System (UIPS) provides a robust and high accuracy positioning [11]. Several ultrasonic sensors are used here. The positions of the ultrasonic sensors are known and fixed. Wi-Fi and RF module are used in each Wireless Sensor Network (WSN). Wi-Fi is used to transfer the data to 
the server. RF module is mainly used for the synchronization of time between different nodes. In order to extract the envelope of beacons of the ultrasonic sensors a new time domain technique is used here to estimate the TOF. Envelope detection filter calculates the position by using Least Square Method(LSM) with the help of sampled values which are on both sides of the envelop. SESKON is a positioning system that uses ultrasonic sensors for locating mobile robots inside buildings [12]. This system uses TDOA method for estimating the position and does not require synchronization between the receiver and transmitter. This system provides a centimeter level accuracy. Three ultrasonic transmitters are used in this system whose coordinates are known and fixed. TELIAMADE is a LPS using wireless ultrasonic sensors [13]. This system provides centimeter level position accuracy. In this system the power consumption is less and has a better network configuration that requires a low memory and low computational cost. RF module is kept on the network mode which helps them to communicate by using the Zigbee protocol. Multilateration is used to calculate the position of mobile node. Angle of incidence limits the location area of the system and location area is also based on the range of sensors. A low cost ultrasonic positioning system is used in [14], where wearable and mobile computers are used to calculate the position. On the ceiling four ultrasonic sensors are kept and are connected to a controller. A radio trigger is send from the controller and an ultrasonic signal is send from the transducer. The difference in the time of arrival of ultrasonic signals is measured by the mobile receiver unit which calculates the position within an accuracy range of $10 \mathrm{~cm}$ and $25 \mathrm{~cm}$.

Scheduling of mobile robot can be done using ultrasonic indoor positioning system [15]. However, ultrasonic sensors are not suitable for large areas. To overcome this, multi-block approach is used which is done by dividing the large areas into several blocks with multiple sensors in each block. Beacon scheduling algorithm is used for estimating the location of the mobile robots. Certain advantages of using this method are that the obstacle problem is reduced to an extent, large areas can be considered for positioning, better placement of sensors is possible, and more mobile robots can be detected. Certain drawbacks include difficulty in recognizing the boundary between each blocks and collision problem. Navigation of mobile robot can be done by using laser range finder, natural landmarks and compass [16]. Corners in the room are considered as natural landmarks here. After detecting the corners, the position of the corners are estimated by using the robot's moving direction which is indicated by the compass. Certain assumptions are made here. i.e. the robots can see the landmarks at all time and no objects are similar to the landmarks in the environment.

Location Based Positioning system using RF and ultrasonic signals based on TDOA give errors [17]. This is due to the lack of Line Of Sight (LOS) of the ultrasonic signals. Therefore to increase the accuracy and to improve the performance a novel TDOA based position estimating technique is used which use ultrasonic reflections in location estimation. This gives a high positioning accuracy and error less than 35 $\mathrm{cm}$ even when the Line Of Sight of signals from the ultrasonic sensors is blocked. Active Bat system used for calculating the orientation and position of objects inside the buildings consist of receivers at known position and they are connected to a PC [18]. RF receiver and ultrasonic transmitters are placed on each rover. An RF message is sent by the PC in every $200 \mathrm{~ms}$. This RF message contains the information about the rover that is going to send the ultrasonic signals. The receiver receives these ultrasonic signals and with the help of multilateration, the position of the rover is calculated. In CRICKET system, the transmitters that are kept on the ceiling of the building emit RF and the ultrasonic signals [19]. RF is placed on the mobile platform which is mainly used for the synchronisation of the transmitters and the receiver. TOA method is used for calculating the position of the mobile unit. The receiver is placed on the mobile unit. This system provides an accuracy of approximately $20 \mathrm{~cm}$. DOLPHIN system is developed to reduce the configuration cost of all sensor nodes [20]. They provide physical location tracking in large areas with better accuracy compared to other systems like Active Bat and Cricket. This system sends and receives ultrasonic and RF signals from the distributed wireless sensor nodes. To different indoor objects, these sensor nodes are attached. This system provides positioning with less manual configuration with the help of a distributed positioning algorithm and with an accuracy of around $15 \mathrm{~cm}$.

However in [14, 18, 19] a narrowband, the ultrasonic sensors used have certain limitations [21]. The first limitation is that the signals can interfere with one another if more than collocated sensors emit signals at the same time. The second limitation is that slow data rates of narrowband systems make it difficult to encode a unique identifier in the short-time ran ging signals. Thus it becomes difficult to separate the signals from different tags for a receiver. In the presence of noise, the system shows poor performance, which is the third limitation. To overcome these problems, broadband ultrasonic transmitters and receivers are used. This system provides certain advantages. The first advantage is noise robustness. That is, the system provides the location updates even in noise. The second advantage is that it provides increased update rates. Broadband ultrasonic systems provide low-latency positioning which is the third advantage. They also provide enhanced identification encoding which is the fourth advantage. 


\section{PROPOSED WORK}

After going through many literature reviews, paper [22] used ultrasonic indoor positioning system called ICKON. The ICKON system estimates the location of the mobile unit at centimetre-level accuracy by using ultrasonic signals. Here the four ultrasonic sensors are placed at known positions and they send signals periodically. Time Difference of Arrival (TDOA) of signals is used by the receiver which is placed on the mobile unit to estimate the location of the mobile unit. Least Square (LS) method is used to solve the set of equations due to its low complexity. Equations formed by using TDOA of signals can be solved by using different methods to obtain the position of the mobile unit based on the complexity and restrictions [23]. They are Analytical Method (AM), Least Square Method (LS), Taylor Series Method (TS), Approximate Maximum Likelihood method (AML), Two-Stage Maximum Likelihood method (TSML) and Genetic Algorithm (GA). Taylor Series method gives less error as compared to other methods. They are used to linearize the non-linear equations [24]. They ignore the measurement errors and provide an accurate result even at noise levels compared to the Least Square method. Therefore in this paper, Taylor Series (TS) method is used as this gives a more accurate result than Least Square (LS) method.

The proposed solution done in this paper to provide a more accurate result in indoor positioning system using mobile robot is to use Taylor Series method in TDOA approach using five sensor coordinates. So far researchers have used Taylor Series method in TDOA approach using four ultrasonic sensors. As the number of transmitters increases the accuracy of the system also increases [23]. The difference between the actual location and the estimated location is reduced to provide a more accurate result.

\subsection{Algorithm}

1. Signals are received from the 5 ultrasonic signals.

2. The Receiver placed on the mobile unit uses TDOA approach to calculate the position.

3. To estimate the position of the mobile unit Taylor Series Method is used [23].

4. We define a function with TDOA estimation with (i+1)th reference node and range estimation error.

$$
\begin{aligned}
f_{i}(x, y, z) & =\sqrt{\left(x-x_{i+1}\right)^{2}+\left(y-y_{i+1}\right)^{2}}-\sqrt{\left(x-x_{1}\right)^{2}+\left(y-y_{1}\right)^{2}} \\
& =m_{i+1,1}+\varepsilon_{i+1,1}
\end{aligned}
$$

where $(x, y)$ is the location coordinate of the mobile unit and $\left(x_{i+1,} y_{i+1}\right)$ is the $(i+1)$ th reference node.

5. The Initial estimation of the mobile unit is taken. Actual location can be written as the sum of initial location and location estimation errors which we have to find out. $\left(\mathrm{x}_{\mathrm{v},} \mathrm{y}_{\mathrm{v}}\right)$ is the initial estimation of the mobile unit. $\delta_{\mathrm{x}}$ and $\delta_{\mathrm{y}}$ are the location estimation errors.

6. Then the function defined in step 5 is expanded into Taylor Series.

$$
f_{i, v}+a_{i, 1} \delta_{x}+a_{i, 2} \delta_{y}=m_{i+1,1}+\varepsilon_{i+1,1}
$$

where $f_{i, v}=f_{i}\left(x_{v}, y_{v}\right)$ and $a_{i, 1}$ and $b_{i, 1}$ are the partial derivatives of the function defined in step 5 with respect to $\mathrm{x}$ and $\mathrm{y}$

7. Equation (2) can be written as

$$
\begin{aligned}
& \mathrm{A} \delta=\mathrm{D}+\mathrm{E}
\end{aligned}
$$

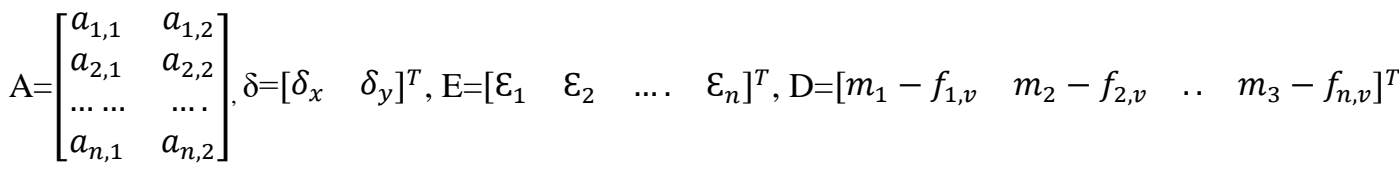

8. Weight Least Square estimation is used to solve this and helps to find location estimation error.

$$
\delta=\left(A^{T} Q^{-1} A\right)^{-1} A^{T} Q^{-1} D
$$

where $\mathrm{Q}=E\left\{\varepsilon \varepsilon^{T}\right\}$, is a covariance matrix with zero-mean Gaussian random variables as elements.

9. Location estimation is continuously updated with the location estimation error.

10. By iterating the above procedures, the location estimation can be continuously refined. 


\section{RESULT AND ANALYSIS}

Matlab was used for simulating the result. The result of the Least Square Method, Taylor Series Method and Analytical Method was obtained using this as shown in Figure 1. A 100 by100 area was taken as the network size. The actual location of the mobile unit was obtained by multiplying the network size and random $(M, 2)$ function where $M$ is taken as the number of mobile units. The number of mobile node is taken as one. The results were also obtained by using four and five sensor nodes using Taylor Series Method which is shown in Table 2 and Table 3.

Root Mean Square Error (RMSE) is the root of sum of square of the difference between the actual location and the estimated location [25, 26]. RMSE obtained in Table1 for Taylor Series Method is $2.11 \mathrm{~m}$, Least Square Method is $38.241 \mathrm{~m}$ and Analytical Method is $27.707 \mathrm{~m}$. This clearly shows that Taylor Series Method provides a closer result to the actual location. That is, Taylor Series method provides an accurate result compared to Least Square method and Analytical Method. So far Taylor Series method in TDOA approach is used with four sensor coordinates. Since the accuracy of the system increases along with increase in number of transmitters, results are shown below using Taylor Series method in TDOA approach with five sensor coordinates.

Table 2 shows the result of Taylor Series Method using four sensor coordinates. Root Mean Square Error (RMSE) obtained for the Taylor Series Method using four sensors is $2.2908 \mathrm{~m}$. Table 3 shows the result of Taylor Series Method using five sensor coordinates.RMSE obtained in using five sensors is $0.764183 \mathrm{~m}$. RMSE is less when five ultrasonic transmitters are used to calculate the position than using four ultrasonic transmitters. This means that a more accurate result is obtained when we use five ultrasonic transmitters rather than four ultrasonic transmitters. Less error is obtained when five ultrasonic transmitter system is used. Figure 1 shows the comparison of error in using 5 sensor coordinates and 4 sensor coordinates. It clearly depicts that error is less when 5 sensor coordinates are used instead of 4 sensor coordinates.

Table 1.The result of TDOA using Least Square Method, Analytical Method and Taylor Series Method

\begin{tabular}{ccccc}
\hline Coordinates & $\begin{array}{c}\text { Actual } \\
\text { Location }(\mathrm{m})\end{array}$ & $\begin{array}{c}\text { Least Square } \\
\text { Method }(\mathrm{m})\end{array}$ & $\begin{array}{c}\text { Taylor Series } \\
\text { Method }(\mathrm{m})\end{array}$ & $\begin{array}{c}\text { Analytical } \\
\text { Method }(\mathrm{m})\end{array}$ \\
\hline$(50,60)(55,70)(90,80)(85,90)$ & $(70.94,75.47)$ & $(68.72,40.82)$ & $(70.63,76.1)$ & $(58.282,58.86)$ \\
$(20,30)(80,65)(20,60)(80,30)$ & $(43.87,38.11)$ & $(20,60.43)$ & $(43.49,38.31)$ & $(38.56,27.554)$ \\
$(30,20)(80,65)(30,60)(80,25)$ & $(56.68,46.98)$ & $(76.71,76.01)$ & $(55.7,47.16)$ & $(44.263,28.828)$ \\
$(40,60)(40,70)(70,90)(70,85)$ & $(27.69,4.617)$ & $(60.187,6.04)$ & $(28.5,4.325)$ & $(62.63,9.785)$ \\
\hline
\end{tabular}

Table 2.The result of TDOA of ultrasonic signals using Taylor Series Method using 4 sensor coordinates

\begin{tabular}{ccc}
\hline 4 Sensor Coordinates & Actual Location $(\mathrm{m})$ & Estimated Location $(\mathrm{m})$ \\
\hline$(20,20)(20,90)(80,30)(80,70)$ & $(67.87,75.77)$ & $(68.49,77.01)$ \\
$(30,60)(50,90)(80,30)(90,70)$ & $(43.87,38.16)$ & $(44.55,37.07)$ \\
$(40,60)(50,80)(90,60)(85,70)$ & $(70.94,75.47)$ & $(70.39,74.18)$ \\
$(50,70)(55,80)(70,90)(85,70)$ & $(81.43,24.35)$ & $(85.89,24.35)$ \\
$(40,70)(45,80)(75,90)(85,80)$ & $(38.04,56.78)$ & $(36.03,58.29)$ \\
\hline
\end{tabular}

Table 3.The result of TDOA of ultrasonic signals using Taylor Series Method using 5 sensor coordinates

\begin{tabular}{ccc}
\hline 5 Sensor Coordinates & Actual Location $(\mathrm{m})$ & Estimated Location $(\mathrm{m})$ \\
\hline$(20,20)(0,70)(20,50)(85,20)(80,90)$ & $(52.11,23.16)$ & $(52.71,23.26)$ \\
$(40,70)(30,50)(35,20)(75,20)(70,90)$ & $(88.52,91.33)$ & $(87.84,91.42)$ \\
$(10,20)(15,70)(20,60)(90,20)(80,80)$ & $(80.55,57.67)$ & $(80.11,56.42)$ \\
$(40,10)(45,70)(40,60)(70,30)(80,80)$ & $(34.39,58.41)$ & $(34.31,57.96)$ \\
$(60,20)(65,70)(50,60)(80,20)(90,80)$ & $(81.81,81.75)$ & $(81.52,82.45)$ \\
\hline
\end{tabular}

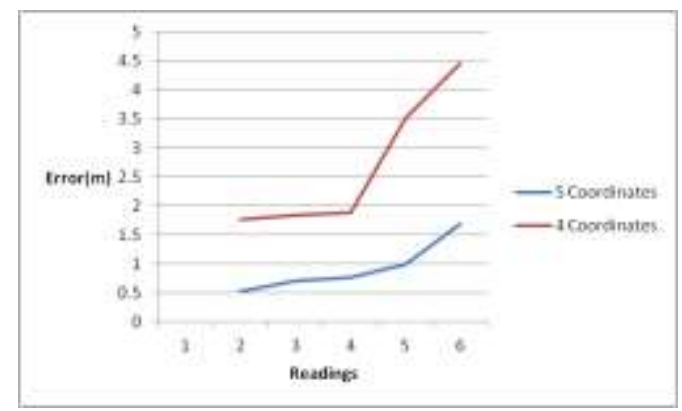

Figure 1. Shows the error comparison in using 5 coordinates and 4 coordinates 


\section{CONCLUSION}

In this paper, the location of the mobile robot was calculated by using an ultrasonic positioning system. The receiver placed on the mobile unit collects the signals from the ultrasonic sensors using TDOA approach. Taylor Series Method in TDOA approach using five ultrasonic sensors is used since this gives a more accurate result compared to the Least Square Method and Analytical Method. The Root Mean Square Error value for Taylor Series Method is less compared to the other two methods. Increasing the number of sensors to five gives an accurate result compared to using four sensors. The Root Mean Square Error value obtained is less while using five sensors compared to using four ultrasonic sensors.

\section{REFERENCES}

[1] S. Al-hadhrami, et al., "Ultra Wideband Indoor Positioning Technologies : Analysis and Recent Advances $\dagger$," pp. 1-36, 2014.

[2] F. Dwiyasa and M. Lim, “A Survey of Problems and Approaches in Wireless-based Indoor Positioning,” pp. 4-7, Oct 2016.

[3] C. Huang, et al., "Real-Time RFID Indoor Positioning System Based on Kalman-Filter Drift Removal and Heron-Bilateration Location Estimation," vol. 64, pp. 728-739, 2015.

[4] P. Kriz, et al., "Low Energy Beacons," vol. 2016, 2016.

[5] U. Of, "WiFi-Based Indoor Positioning," pp. 150-157, Mar 2015.

[6] A. Yazici, et al., “An Ultrasonic Based Indoor Positioning System,” pp. 585-589, 2011.

[7] M. S. Rahman and K. Kim, "Indoor Positioning by LED Visible Light Communication and Image Sensors," vol. 1, pp. 161-170, 2011.

[8] Y. Gu, et al., "Wireless Personal Networks," vol. 11, pp. 13-32, 2009.

[9] M. Farooq-i-azam, "Location and Position Estimation in Wireless Sensor Networks," pp. 1-52.

[10] J. Li, et al., "An Indoor Ultrasonic Positioning System Based on TOA for Internet of Things," vol. 2016, 2016.

[11] J. Qi and G. Liu, "A Robust High-Accuracy Ultrasound Indoor,” 2017.

[12] A. Yazici, et al., “An Ultrasonic Based Indoor Positioning System,” pp. 585-589, 2011.

[13] S. Network, "A Robust High-Accuracy Ultrasound Indoor," 2017.

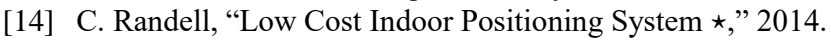

[15] R. S. Nair, "Scheduling of Indoor Mobile Robot using Ultrasonic," vol. 1, pp. 8-12, 2014.

[16] O. Parlaktuna, et al., "Localization of a Mobile Robot using Natural Landmarks and Sensor Fusion," vol. 26480, pp. 3-6.

[17] K. Kim, et al., "Ultrasonic Reflections," pp. 1-14, 2016.

[18] A. Ward, et al., "A New Location Technique for the Active Office."

[19] S. M. C. Science, et al., "The Cricket Indoor Location System," 2005.

[20] Y. Fukuju, et al., "Dolphin: An autonomous indoor positioning system in ubiquitous computing environment," in Proc. of the IEEE Workshop on Software Technologies for Future Embedded Systems. Washington, DC, USA: IEEE Computer Society, pp. 53-57, 2003.

[21] S. M. C. Science, et al., "The Cricket Indoor Location System," 2005.

[22] U. Yayan, et al., "A Low Cost Ultrasonic Based Positioning System for the Indoor Navigation of Mobile Robots," pp. 541-552, 2015.

[23] G. Shen, et al., "Performance Comparison of TOA and TDOA Based Location Estimation Algorithms in LOS Environment," vol. 2008, 2008.

[24] X. Li, et al., "Contributed Review : Source-localization algorithms and applications using time of arrival and time difference of arrival measurements Contributed Review: Source-localization algorithms and applications using time of arrival and time difference of arrival measurements," vol. 41502, 2016.

[25] Y. R. Hamdy and S. A. Mawjoud, "Performance Assessment of U-TDOA and A-GPS Positioning Methods," pp. 99-104, 2012.

[26] E. J. Nnenna and O. H. Onyekachi, "Mobile Positioning Techniques in GSM Cellular Networks : A Comparative Performance Analysis," vol. 2, pp. 21-29, 2012.

\section{BIOGRAPHIES OF AUTHORS}

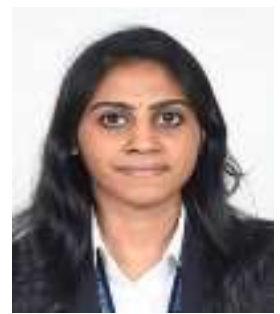

Diniya Jose persuing the MCA degree from CHRIST ( Deemed to beUniversity),India. She had received degree from St.Xaviers College for Women, Aluva.Her research interest is Indoor Positioning System.

E-mail:diniya.jose@mca.christuniversity.in 


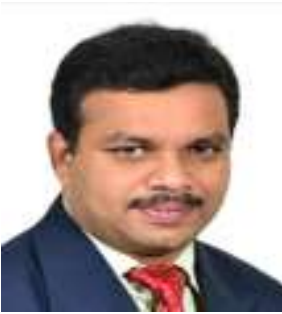

Shoney Sebastian received MPhil degree from Vinayaka Missions University in 2007.MCA degree from Indira Gandhi National Open University in 2000.PGDCA degree from Institute of Human Resource Development for Electrionics in 1995 and now pursuing $\mathrm{PhD}$ degree from CHRIST (Deemed to be University).Currently, he is working as the Associate Professor in CHRIST (Deemed to be University).His research interest includes cloud computing, data mining and Indoor Positioning system.

E-mail:shoney.sebastian@christuniversity.in 